\title{
TAGUNG
}

\section{Die EU als strategischer Akteur? Die Außenpolitik der EU auf dem Prüfstand}

\author{
Marnie Schoeller*
}

Die Europäische Union wird oft als komplexes, ja kompliziertes Konstrukt charakterisiert. Die Außenpolitik bildet dabei keine Ausnahme es gibt politische Ansätze für die Nachbarschaft, für die Erweiterung, für einzelne Partnerschaften und Integration verschiedener Politikfelder. Was bezweckt die Europäische Union mit dieser Variation an außenpolitischen Maßnahmen und wie effizient sind diese tatsächlich?

Die Komplexität des Themas nahm die Europäische Akademie Berlin gemeinsam mit dem Arbeitskreis Europäische Integration zum Anlass, eine Konferenz über die Grenzen der Integration und Europas Ansätze für die Nachbarschaft zu organisieren. Verschiedene Vorträge veranschaulichten das System der europäischen Außenpolitik, evaluierten, kritisierten und kommentierten die umfassenden Strategien der Europäischen Union.

\section{Die Ziele der Europäischen Nachbarschaftspo- litik}

Zum Auftakt gab Madeleine Majorenko einen kurzen Rückblick über die Entstehung und die Notwendigkeit der Europäischen Nachbarschaftspolitik (ENP). Im Jahr 2004 wurde die ENP gegründet, basierend auf der Erkenntnis, dass es im aufgeklärten Selbstinteresse der Europäischen Union sei, gute Beziehungen mit den Nachbarstaaten zu haben. Die ENP sei jedoch kein Weg zur Erweiterung; die Perspektive einer EU-Mitgliedschaft habe es für die ENP-Staaten von Beginn an nicht gegeben.

\section{Europas strategische Ansätze für die Nachbarregionen}

Tagung der Europäischen Akademie Berlin in Kooperation mit dem Arbeitskreis Europäische Integration, mitfinanziert durch die Europäische Union innerhalb des „Programms Lebenslanges Lernen“

Mit freundlicher Unterstützung des Auswärtigen Amtes

\section{3.-25. Januar 2013, Berlin}

Das Arbeitsprogramm der Europäischen Kommission 2013 im Hinblick auf die Aktivitäten in der Nachbarschaft der EU Madeleine MAJORENKO, Europäischer Auswärtiger Dienst, Brüssel

Europas Instrumentenkasten: Partnerschaften, Strategien, Synergien - ein Überblick

Prof. Dr. Eckart D. STRATENSCHULTE, Leiter, Europäische Akademie Berlin

Baustelle Balkan: Die Erweiterungspolitik der EU zwischen Erfolg und Stillstand

Dr. Andrea DESPOT, stellvertretende Leiterin, Europäische Akademie Berlin

Sackgasse nach Ankara? - Die Beitrittsverhandlungen der EU mit der Türkei

Dr. Andreas MARCHETTI, Rheinische FriedrichWilhelms-Universität, Zentrum für Europäische Integrationsforschung, Bonn

One Size Fits All? - Die Europäische Nachbarschaftspolitik im Spagat zwischen Ost und Süd

Dr. Julia LANGBEIN, Freie Universität Berlin

\footnotetext{
* Marnie Schoeller, BA in European Studies, Projektassistentin, Europäische Akademie Berlin.
} 
Kernelemente der ENP seien bilaterale Aktionspläne, mit deren Hilfe die Europäische Union und die einzelnen Partnerstaaten eine Art Agenda für anstehende politische und wirtschaftliche Reformen entwickeln.

Durch den Arabischen Frühling wurde die ENP auf die Probe gestellt. Es sei deutlich geworden, dass der, one size fits all'-Ansatz nicht zufriedenstellend sei, da jeder Staat sich unterschiedlich schnell verändere und andere Bedürfnisse habe, auf die die Partnerschaft eingehen müsse. Deswegen sei ein maßgeschneiderter Ansatz wichtig, der sich in dem Prinzip ,mehr für mehr' widerspiegle, das heißt je mehr die betroffenen Staaten sich reformieren und verändern, desto mehr werden sie dafür auch belohnt. Majorenko sieht das kommende Gipfeltreffen der Östlichen Partnerschaft im November 2013 in Vilnius als potenziell wegweisend an. Es werde dabei besonders darum gehen, ob die Assoziierungsabkommen mit der Republik Moldau und der Ukraine unterschrieben werden. Sie selbst sprach sich für eine Unterzeichnung aus. Es solle nicht vernachlässigt werden, dass diese östlichen Staaten zu Europa gehören und dass auch Russland erhebliches Interesse an ihnen habe. Sie stellte heraus, dass die weitere Entwicklung von der Verabschiedung des mehrjährigen Finanzrahmens der Europäischen Union (2014-2020) abhinge, da in diesem festlegt wird, wie viel Geld für die Nachbarschaftspolitik zu Verfügung stehen werde.

In seinem Vortrag fasste Eckart D. Stratenschulte die Vielfalt der Strategien zusammen, die der Europäischen Union in ihrer Kooperation mit anderen Staaten zur Verfügung stehen. Eine Strategie sei dadurch definiert, dass sie ein Ziel habe, dessen Erreichen jedoch durch Hindernisse und Widerstände erschwert werde. Die gemeinsamen Ziele der Europäischen Union, so Stratenschulte, seien Stabilität, Demokratieförderung und Prosperität. Basierend auf der Überzeugung, dass es nur möglich sei, in Frieden zu leben solange man stabile und friedliche Nachbarn habe, verfolge die Europäische Union diese Ziele ebenfalls in ihrer
Sandkastenspiele im Land der Zwerge?

Europas Nachbarschaftsstrategien im Lichte der globalen Entwicklung

Prof. Dr. Hanns W. MAULL, Universität Trier

Ahnungslos? Die Union für das Mittelmeer und der arabische Frühling

Prof. Dr. Annette JÜNEMANN, Helmut-SchmidtUniversität, Hamburg

Synergie ohne Effekt? Die Schwarzmeerstrategie der $\mathbf{E U}$

Dr. David KERESELIDZE, Botschafter a.D., stellvertretender Direktor, International Centre for Black Sea Studies, Istanbul

Europas Tankstelle oder Russlands Hinterhof? Zentralasien im strategischen Fokus der EU

Hans-Jochen Schmidt, Botschafter a.D.

Partner oder Störfaktor: Russlands Rolle in der europäischen Nachbarschaft

Prof. Dr. Hannes ADOMEIT, Professor für Osteuropastudien, Europakolleg, Brügge

Uncle Sam in Europe: Die Europapolitik der USA

Dr. Henning RIECKE, Deutsche Gesellschaft für Auswärtige Politik, Berlin

Ratlos? Die Östliche Partnerschaft der Europäischen Union

Dr. Weronika PRIESMEYER-TKOCZ, Studienleiterin, Europäische Akademie Berlin

(un-)mittelbaren Nachbarschaft. Dafür stünden der Europäischen Union verschiedene Instrumente zur Verfügung. Im Folgenden differenzierte Stratenschulte diesen ,Instrumentenkasten", indem er die verschiedenen strategischen Ansätze darstellte und unterschiedliche Strategietypen herausarbeitete.

Zuerst nannte er die Erweiterungsstrategie, die als Stabilisierungsmaßnahme fungiere. Sie resultiere in einer vollen Übernahme des acquis communautaire und biete volle Mitwirkungsmöglichkeiten für die betroffenen Staaten. Er nannte die Assoziierungsstrategie, die eine 
weitgehende Übernahme des acquis und nur eingeschränkte Mitwirkungsmöglichkeiten vorsehe. Die ENP, die Östliche Partnerschaft sowie die Union für das Mittelmeer seien Assoziierungsstrategien, sie böten ,everything but institutions“. Ein dritter Strategietyp sei die Entwicklungsstrategie. Laut Stratenschulte böte die Strategie unterschiedliche Mitwirkungsmöglichkeiten, je nachdem ob ein Staat EUMitglied sei oder nicht. Als Beispiele nannte er die Donaustrategie, die Ostseestrategie sowie die Schwarzmeersynergie. Ein weiterer Strategietyp sei die Partnerschaftsstrategie, die auf eine Veränderung des Partners abziele und gleichzeitig eigene Interessen verfolge. Dies sei beispielsweise an der Zentralasienstrategie zu erkennen.

Es gebe Staaten, die im Rahmen von mehreren Partnerschaften mit der Europäischen Union kooperieren. Stratenschulte warf daher die Frage nach einer Neuordnung des Instrumentenkastens auf.

\section{Die Bedeutung der Beitrittsperspektive für die westlichen Balkanstaaten und die Türkei}

Die Erweiterungspolitik der Europäischen Union genauer unter die Lupe nehmend, beschäftigte sich Andrea Despot mit den sogenannten westlichen Balkanstaaten Albanien, Bosnien und Herzegowina, Kosovo, Ehemalige Jugoslawische Republik Mazedonien, Montenegro, Serbien sowie Kroatien. Bis 1995/1999 ein Raum des Kriegs, des Unrechts und der Instabilität, seien diese Staaten Nachzügler im Transformationsprozess (nach dem Zerfall der Sowjetunion) gewesen - wirtschaftlich, politisch sowie gesellschaftlich. Der Europäische Rat habe 1998 den Westbalkan als nächstes strategisches Erweiterungsziel definiert und seit 2001 seien mit den Balkanstaaten Stabilisierungs- und Assoziierungsabkommen geschlossen worden. Des Weiteren habe der Europäische Rat sowohl 2003 als auch 2006 die Beitrittsperspektiven der Staaten des Balkans bekräftigt. Diese Entwicklungen hätten bei den Balkanstaaten große Erwartungen auf einen absehbaren EU-Beitritt geweckt. Die Staaten sähen sich als ,integraler Bestandteil Europas" und fühlten sich zum europäischen Kulturraum zugehörig. Die Europäisierung der westlichen Balkanstaaten habe als Friedensanker, Reformmotor und Stabilisierungsinstrument gewirkt. Funktioniert habe dies, weil Reformmaßnahmen seitens der Balkanstaaten mit greifbaren Beitrittsperspektiven honoriert worden seien. Aktuelle Trends und Krisen innerhalb der Europäischen Union veränderten die Situation für die westlichen Balkanstaaten ungemein. Aufgrund der Diskussion von Desintegrationsszenarien Europas, einer sich ausbreitenden Erweiterungslethargie sowie der begrenzten institutionellen und finanziellen Absorptionsfähigkeit der Europäischen Union habe sich in den westlichen Balkanstaaten ein Wandel von „EU-phorie“ zu „EU-Realismus“ vollzogen. Nach dem EU-Beitritt Kroatiens sei ein faktischer Erweiterungsstopp für diese Region zu erwarten. Despot betonte, dass die strategische Relevanz der westlichen Balkanregion fortbestünde. Aus diesem Grund sprach sie sich für weitere Schritte auf dem Weg zu einer EU-Mitgliedschaft aus. Neben weiterhin existierenden ethno-nationalen Spannungen gebe es immer noch ungelöste Status- und Territorialkonflikte, für deren Moderation die Europäische Union in der politischen Verantwortung stünde. Ohne eine realistische EU-Option würden sich sowohl die Suche nach alternativen Entwicklungsmodellen als auch die politische Neuorientierung der Staaten auf dem Westbalkan verstärken. Es sei daher essenziell, Ziele eindeutig zu bestimmen, um der westlichen Balkanregion eine faire und glaubhafte Perspektive zu bieten.

Andreas Marchetti widmete seinen Vortrag den Beitrittsverhandlungen mit der Türkei. Bereits im Jahre 1987 hat die Türkei den Antrag auf Mitgliedschaft gestellt. Nachdem 1995 eine Zollunion zwischen der Europäischen Union und der Türkei geschlossen worden ist, wurde 2005 schließlich mit den Beitrittsverhandlungen begonnen. Verschiedene Aspekte verkomplizierten diese. Zum einen seien die Ein- 
flussmöglichkeiten der einzelnen Mitgliedstaaten der Europäischen Union auf den Erweiterungsprozess sehr groß. Zum anderen herrsche derzeit eine Erweiterungsmüdigkeit innerhalb der Europäischen Union. Bei den Beitrittsverhandlungen mit der Türkei herrsche faktisch Stillstand. Marchetti betonte, dass die Sicherheit eines Beitrittes aus Sicht der Türkei nicht mehr gegeben sei. Die Europäische Union wirke unglaubwürdig und fahre eine „Hinhaltetaktik“. In der Folge orientiere sich die Türkei politisch anders. Ihre aktive Politik richte sich zunehmend auf den Kaukasus, die Balkanregion und den Nahen Osten. Marchetti kritisierte, dass die Europäische Union nicht fähig sei als strategischer Akteur zu handeln. Sie sei vielmehr ein semistrategischer Akteur. Die Europäische Union habe zwar klare Zielsetzungen, leide jedoch unter einer fehlenden Priorisierung. Dem semistrategischen Charakter lägen verschiedene Ursachen zugrunde. Zum einen gebe es ungünstige Rahmenbedingungen, wie die europäische Staatsschuldenkrise, die Persistenz nationaler Vorbehalte und Unklarheit über die ,Finalität der Union. Zum anderen gebe es innerhalb der Europäischen Union strukturelle Spannungen. Diese äußerten sich beispielsweise in dem Konflikt zwischen dem Eintreten für Normen und der Verfolgung von Interessen. Ebenso gebe es Kontraste zwischen Ambitionen und Fähigkeiten der Staatengemeinde sowie zwischen den Konzepten der Intervention und der Verteidigung. Er glaube an ein weiterhin bestehendes Interesse der Türkei an einer intensiveren politischen Einbindung in die Europäische Union. Allerdings müsse die politische Kommunikation verbessert werden, um öffentliche Akzeptanz und Verständnis für die Bedeutung der Einbeziehung der Türkei zu erreichen.

Im folgenden Vortrag beschäftigte sich Julia Langbein mit der Europäischen Nachbarschaftspolitik (ENP) und erörterte, ob es sich dabei um eine differenzierte Strategie oder eine ,one size fits all'-Politik handelt. Bevor sie auf die erneuerte ENP einging, lieferte sie eine kri- tische Beleuchtung der „alten ENP“ (2004 bis 2010). Ein Problem der ,alten ENP“sei die Gegensätzlichkeit der Partner gewesen, die teilweise willig, teilweise zögerlich oder gar resistent gewesen seien. Langbein bemängelte eine fehlende Antwort der Europäischen Union auf problematische Regionen und fehlende klare Zielsetzungen. Dennoch seien die Probleme in diesen Staaten oft ähnlich gewesen: Korruption, fehlende Bildung, schwache Wirtschaft und mangelnde Demokratie. Deshalb sei es möglich gewesen, gemeinsame Probleme im Rahmen der ENP zu adressieren. Es habe zwar länderspezifische Überwachungen der Reformen gegeben, dennoch seien die Handlungsanweisungen begrenzt gewesen. Letztendlich stellte sie dar, dass die ,alte ENP“"zwar kein ,one size fits all'-Ansatz gewesen sei, aber die Differenzierung zwischen den einzelnen Staaten häufig nicht ausreichend und teilweise sogar kontraproduktiv gewesen sei. Die erneuerte ENP (2011/2012) sehe vor, dass mittelund langfristige Prioritäten der Zusammenarbeit klarer definiert werden. Die Fortschrittsberichte, die 2012 veröffentlicht wurden, seien dennoch immer noch relativ schwammig formuliert. Der neue Ansatz der stärker differenzierten Konditionalität gründe sich auf der Formel ,mehr für mehr'. Dennoch seien politikfeldspezifische Anreize notwendig. Die neue ENP beinhalte, dass Fortschrittsberichte nun auch Handlungsanweisungen geben. Weiterhin umfasse die neue ENP eine gezielte Förderung der Zivilgesellschaft und wirtschaftlicher Akteure. Zusammenfassend betonte Langbein, dass die neue ENP viele Kritikpunkte aufgreife. Dennoch gebe es weiterhin undeutliche Orientierungswerte, besonders im Bereich der Demokratieförderung. Es sei beispielsweise immer noch unklar, welche Nichtregierungsorganisationen gefördert werden sollen. Dass entscheidende Akteure vor Ort schlichtweg unbekannt seien, erschwere das Einschätzen von Zielen, Motiven und Absichten der potenziellen Partnern und somit auch eine Zusammenarbeit. 
Europas Außenpolitik im globalen Zusammenhang

Hanns W. Maull beleuchtete die europäischen Bemühungen in der Nachbarschaft im Lichte der globalen Entwicklung. Nach 1990 sei die Politik der konzentrischen Kreise unzureichend gewesen, sodass spätestens mit der Entwicklung der Kopenhagener Kriterien 1993 die Weichen für eine Erweiterung der Union gestellt wurden. Folgerichtig gab es 1995 und 2004 beziehungsweise 2007 zwei große Erweiterungswellen. Diese seien - abgesehen von kleinen Mängeln - außerordentlich erfolgreiche Stabilisierungs- und Transformationsmechanismen gewesen. Als Mitte der 1990er Jahre der Balkankrieg tobte, habe sich das dramatische Versagen der Europäischen Union als Krisenmanager gezeigt. Nachdem die USA keinerlei Interesse an der Mediation dieser Konflikte gezeigt habe, seien NATO-Interventionen ${ }^{1}$ für die Befriedung des ehemaligen Jugoslawiens notwendig gewesen. In den Staaten der Östlichen Partnerschaft stünden die Beitrittswünsche etlicher osteuropäischer Staaten dem Erweiterungsüberdruss in der Europäischen Union gegenüber. Die Politik der Europäischen Union gegenüber dem südlichen Mittelmeerraum fände ihren Rahmen im Barcelona Prozess und der Union für das Mittelmeer. Die Zielsetzungen Transformation und nachhaltige Entwicklung seien jedoch in den Hintergrund getreten, während Sicherheit und Stabilität zunehmend dominierten. Maull betonte, dass die Europäische Union kein „Zwerg“ sei und schon gar nicht im „Land der Zwerge“ agiere. Sie habe durchaus die Mittel, um auf ihre Nachbarschaft Einfluss zu nehmen. Was fehle sei ausschließlich eine plausible Strategie. Er beschrieb, dass die Europäische Union derzeit strukturell strategieunfähig schiene und begründete dies mit der Art und Weise, wie Entscheidungen zustande kämen. Maull betonte, dass die Interessen eines Akteurs immer auch dessen Wertevorstellungen reflektierten. Das Problem sei jedoch, dass sich in politi- schen Prozessen kurzfristige Interessen durchsetzten. Er wies auf die Möglichkeit hin, dass die europäische Integration am Ende ihrer transformativen Möglichkeiten angekommen sein könnte. Er betonte, dass das Prinzip der Subsidiarität ernst genommen werden solle. Nötig sei eine klare Prioritätensetzung, eine Begrenzung auf einige wenige bedeutsame, vielversprechende Zielsetzungen und auch eine Reduzierung der Instrumente.

\section{Die Bedeutung des Arabischen Frühlings und die Bedeutungslosigkeit der Union für das Mittelmeer}

In ihrem Vortrag setzte sich Annette Jünemann kritisch mit der Union für das Mittelmeer (UfM) und dem Arabischen Frühling auseinander. Die Genese der UfM 2007/2008 sei ein persönliches Projekt des damaligen französischen Präsidentschaftskandidaten Nicolas Sarkozy gewesen. Die Europäische Union sei eine Partnerschaft mit Autokratien eingegangen, sodass Themen wie Demokratieförderung und Menschenrechte nicht mehr thematisiert worden seien. Dadurch wurde die normative Dimension faktisch aufgegeben. Die Europäische Union habe dies im Zuge der ,Versicherheitlichung" in Kauf genommen, da man sich im Kampf gegen den Terror befunden habe. Der Arabische Frühling habe die Europäische Union überrascht und werfe nun ein grelles Licht auf das normative und realpolitische Versagen der Staatengemeinschaft in ihrer Politik gegenüber den südlichen Nachbarstaaten. Die Tatsache, dass der Arabische Frühling für die Europäische Union nicht voraussehbar war, läge an verschiedenen Fehleinschätzungen. Die erste sei die fälschliche Annahme gewesen, dass arabische Autokratien stabil seien. Jünemann erklärte, dass es zwar Herrscherwechsel aber keineswegs Systemwechsel gab. Die autokratischen Regime seien vielmehr anpassungsfähig gewesen und hätten rechtzeitig auf Entwicklungen innerhalb der Bevölkerung reagiert (,authoritarian learning ${ }^{`}$ ). Des Weiteren

1 North Atlantic Treaty Organization. 
habe es innerhalb der Europäischen Union mangelnde Kenntnisse der gesellschaftlichen Strukturen der nordafrikanischen Länder gegeben. Jünemann stellte die Reaktionen der Europäischen Union auf den Arabischen Frühling kritisch dar. Zu Beginn der Proteste sei die Situation komplett verkannt und falsch eingeschätzt worden. Es habe weder eine einheitliche europäische Linie noch eine zeitnahe eindeutige Reaktion gegeben (,too little too late'). Des Weiteren habe die UfM im Arabischen Frühling überhaupt keine Rolle gespielt. Im Zuge der aktuellen Entwicklung sei es nun besonders wichtig, die Glaubwürdigkeit der Europäischen Union wieder aufzubauen.

\section{Problematische Beziehungen zum}

Schwarzmeerraum und Zentralasien

David Kereselidze stellte in seinem Vortrag die Schwarzmeersynergie vor, die 2008 in Kraft trat und sich an die Schwarzmeerregion ${ }^{2}$ richtet. Die Ziele der Schwarzmeersynergie seien von Beginn an vielfältig gewesen. Die Kooperation mit und zwischen den Schwarzmeerstaaten sollte intensiviert werden, demokratische und wirtschaftliche Reformen sowie Stabilität und Entwicklung unterstützt werden. In sich sei diese Region politisch heterogen, sie habe große Probleme im Bereich des Aufbaus demokratischer Strukturen, regionaler Kooperation und der Beilegung regionaler Konflikte. Die Schwarzmeersynergie habe sehr hohe Erwartungen bei den Mitgliedstaaten geweckt, da sie die erste Form einer Kooperation mit der Europäischen Union gewesen sei. Sie habe verschiedene wichtige politische Partner zusammengebracht und adressiere gemeinsame Probleme: Transport, Energie, Umwelt und Wirtschaft. Problematisch sei jedoch, dass es viele Hindernisse gebe und einige Staaten sehr widerwillig gegenüber einer Zusammenarbeit mit der Europäischen Union seien. Die, frozen conflicts ' der Region blockierten viele Projekte und das Thema Energie führe zu vielen Kon- flikten. Die Schwarzmeersynergie stelle keine Priorität innerhalb der Europäischen Union dar. Daraus resultierten sehr begrenzte Fortschritte, besonders in den Bereichen Demokratie, Handel und Konfliktlösung. Kereselidze stellte dar, dass die Schwarzmeersynergie in den letzten Jahren relativ passiv und erfolglos gewesen sei. Dennoch forderte er weitergehende regionale Kooperation und Integration, die Zivilgesellschaft solle mit eingebunden werden und bilaterale Abkommen zwischen der Europäischen Union und den Schwarzmeerstaaten geschlossen werden. Die Synergie solle sich auf eine kleine Anzahl von Prioritäten mit gemeinsamen Interessen fokussieren.

Die Beziehungen der Europäischen Union zu Zentralasien standen im Mittelpunkt des Vortrags von Hans-Jochen Schmidt. Er beschrieb, dass sich auch dort ganz deutlich der Konflikt zwischen der Wahrung der Interessen auf der einen und dem Eintreten für Werte auf der anderen Seite zeigt. Oft würden die grundlegenden Werte der Europäischen Union durch diesen Konflikt in den Hintergrund gedrängt. Unter der deutschen EU-Ratspräsidentschaft 2007 wurde die Zentralasienstrategie verabschiedet. Erstmals seien politische Leitlinien für ein größeres Engagement der Europäischen Union mit Kasachstan, Kirgistan, Tadschikistan, Turkmenistan sowie Usbekistan formuliert worden. Schmidt verwies auf die konstant wachsende strategische Bedeutung, die diese Region für die Europäische Union habe. Eine regionale Kooperation und eine grenzübergreifende Zusammenarbeit zwischen den Staaten in Zentralasien seien auch im Interesse der Europäischen Union. Neben dem großen Thema der Energieversorgung und anderen Rohstoffvorkommen, beschäftige sich die Europäische Union auch mit organisiertem Verbrechen, Drogenhandel, Terrorismus und illegaler Migration. Die Nachbarschaft der Region zu Afghanistan sei ein weiterer wichtiger Aspekt. Schmidt betonte, dass die Konditionalität ge-

2 Armenien, Aserbaidschan, Georgien, Republik Moldau, Russland, Türkei, Ukraine sowie die EU-Staaten Griechenland, Bulgarien und Rumänien. 
genüber den Staaten der zentralasiatischen Region zielorientierter sein müsse. Es sei notwendig, die Situation der Menschen in diesen Staaten grundlegend zu verbessern, denn erst dann könne man sie von den Werten und Prinzipien der Europäischen Union überzeugen. Ein Schwerpunkt der Zentralasienstrategie sei daher der Aufbau rechtsstaatlicher Strukturen und die Achtung der Menschenrechte.

\section{Russland als Störfaktor der europäischen Außenpolitik?}

Auch Russland hat Einfluss auf die europäischen Ansätze in der Nachbarschaft der Europäischen Union. Hannes Adomeit vertrat in seinem Vortrag die Position, dass Russland weiterhin ein Störfaktor in der europäischen Nachbarschaft bleibt. Russlands Politik in den Staaten der $\mathrm{GUS}^{3}$-sei eine Verlängerung der russischen Innenpolitik; Russland versuche, die eigenen Überzeugungen und Ordnungen auf diese Staaten zu übertragen. Dabei sei die russische Politik von folgenden Strukturelementen geprägt: die Konzentration der Macht auf einen kleinen Kreis, mangelnde Transparenz, populistischer Führungsstil, Wahlmanipulation, fehlende Gewaltenteilung, staatliche Kontrolle über die Wirtschaft, Korruption, politische Kontrolle der Judikative und eine eingeschränkte Medienfreiheit. Russlands Pfad unter Wladimir Putin widerspräche dabei allen Werten, die der Europäischen Union zugrunde liegen: pluralistische Demokratie, Rechtsstaat, eine prosperierende freie Marktwirtschaft und eine aktive Zivilgesellschaft. Dadurch werde die Kooperation enorm erschwert. Ferner versuche Russland, die EU-Integrationsbemühungen gegenüber den GUS-Staaten abzuwenden und biete „Integrationskonkurrenz“. Die Wirtschafts- sowie Energiepolitik Russlands seien dabei die wichtigsten Instrumente, um eine Annäherung der Staaten an die Europäische Union zu verhindern. In Bezug auf die,frozen conflicts' in der Region hätten sich Russland und die Europäische Union dazu verpflichtet, gemeinsame Ansätze zur Lösung dieser Konflikte zu schaffen. Dies sei jedoch nicht geschehen; im Gegenteil: Putins Merkmal im Nachbarschaftsraum sei vielmehr die Instrumentalisierung der Konflikte als deren Lösung. Adomeit betonte, dass es höchst unwahrscheinlich sei, dass sich Russlands Haltung gegenüber der Europäischen Union ändere. Aus Russlands Sicht sei diese Kooperation ein Nullsummen-Verhältnis und keineswegs eine Win-win-Situation.

\section{Die Bedeutung der transatlantischen Kooperation}

Die Europapolitik der USA war das Thema des Vortrags von Henning Riecke. Er beschrieb, dass die europäische Integration ohne den USSchutzschirm während des Kalten Kriegs niemals zustande gekommen wäre. Das amerikanische Verhältnis zur europäischen Integration sei gleichsam geprägt von Unterstützung und gespannter Aufmerksamkeit. Nach dem Ende des Ost-West-Konflikts habe es einige Spannungen und grundsätzlich verschiedene Ansätze zwischen Amerikanern und Europäern gegeben. Dazu gehöre die unterschiedliche Einstellung zum Völkerrecht sowie zu multilateralen Organisationen, die seitens der Europäischen Union besonders wertgeschätzt würden. Bei der Anwendung von militärischer Gewalt habe die Europäische Union darauf bestanden, dass sie lediglich ein letztes Mittel sei. Während die USA sich als Supermacht gesehen hätten, die überall ihre Werte vertreten wolle, sei Europa eher auf sich selbst und seine Nachbarschaft fokussiert gewesen. Durch die neuen Machtschwerpunkte habe es nun zwar eine strategische Umorientierung seitens der USA gegeben, jedoch keineswegs ein Abwenden von Europa. Riecke betonte, dass es keinen ,Asian shift' gegeben habe. Im Gegenteil, die Europäische Union bliebe für die USA sehr relevant. Vielmehr habe sich das Selbstbild der

3 Gemeinschaft unabhängiger Staaten: Armenien, Aserbaidschan, Belarus, Kasachstan, Kirgistan, Republik Moldau, Russland, Tadschikistan, Turkmenistan, Ukraine und Usbekistan. 
USA verändert (besonders unter Präsident Barack Obama). Die Amerikaner hätten realisiert, dass sie ihre eigene Wirtschaftsleistung erhöhen müssten, um außenpolitisch aktiver zu sein und würden sich daher auf ihre eigenen, nationalen Probleme besinnen (,nation-building at home"). Riecke wies auf gemeinsame Aufgaben für die USA und die Europäische Union hin. Es sei wichtig, eine gemeinsame transatlantische Position zur Asien-Pazifik-Region zu vertreten. Darüber hinaus solle eine stabile Situation in Nordafrika und im Mittleren Osten erreicht werden. In Zeiten der Schwäche der USA durch wirtschaftliche Probleme und einen signifikanten Einflussverlust habe die Europäische Union, so Riecke, die Gelegenheit, ihren Einfluss in der Nachbarschaft zu stärken. Er sei sich jedoch „fast sicher, dass die Europäische Union diese Chance verpassen wird“.

\section{Wie effektiv ist die Östliche Partnerschaft?}

Weronika Priesmeyer-Tkocz widmete sich der 2009 in Kraft getretenen Östlichen Partnerschaft, die Teil der Europäischen Nachbarschaftspolitik ist. Ziel sei eine Annäherung der Staaten Armenien, Aserbaidschan, Belarus, Georgien, der Republik Moldau und der Ukraine an die Europäische Union. Sie erklärte, die Partnerschaft resultiere aus dem Wunsch, einen politischen Ansatz gegenüber den osteuropäischen Staaten zu entwickeln. Die deutsche EU-Ratspräsidentschaft (2007) habe dabei als Motivator für die osteuropäische Politik fungiert. Sie habe zwischen den sogenannten Nachbarn Europas (den südlichen Mittelmeerstaaten) und den europäischen Nachbarn unterschieden. Erst nachdem die französischen Interessen durch die Etablierung der Union für das Mittelmeer befriedigt wurden, habe die polnisch-schwedische Initiative einer Östlichen Partnerschaft durchgesetzt werden können. Kritisch sei zu sehen, dass es die EU-Staaten waren, die zunächst unter sich über die Östliche Partnerschaft gesprochen haben. Erst nachdem alle Mitgliedstaaten zugestimmt hätten, seien schließlich auch die betroffenen osteuropäischen Staaten gefragt worden. Wie die bereits existierende ENP biete die Östliche Partnerschaft weitere Impulse für die bilaterale Zusammenarbeit zwischen der Europäischen Union und den östlichen Partnerstaaten. Diese Ebene ermögliche vertiefte vertragliche Beziehungen sowie Mobilität, Visa-Liberalisierung und sektorale Zusammenarbeit. Zusätzlich biete die Östliche Partnerschaft die Möglichkeit für eine multilaterale Zusammenarbeit und eine begrenzte Kooperation mit Belarus, die im Rahmen der ENP nicht stattfinden könnten. Auf der multilateralen Ebene gebe es vier Plattformen, die durch regelmäßige Gipfeltreffen institutionalisiert worden seien. Diese Plattformen beschäftigten sich mit Demokratie, Stabilität und ,good governance' sowie mit der wirtschaftlichen Entwicklung, der Energiesicherheit und der Zivilgesellschaft. PriesmeyerTkocz evaluierte das Konzept der Östlichen Partnerschaft und wies dabei sowohl auf Fortschritte als auch auf Kritikpunkte hin. Verbesserungen, die die Östliche Partnerschaft zusätzlich zur ENP bewirke, seien ein verstärktes Differenzierungsprinzip zwischen den unterschiedlichen Staaten sowie ein Prozess der Sozialisierung durch die Europäisierung der betroffenen Staaten. Das neue Konditionalitätsprinzip ,mehr für mehr' sei durchaus glaubhafter und der neue Zwei-Ebenen-Ansatz (Kooperation auf bilateraler sowie multilateraler Ebene) stelle ebenfalls einen positiven Aspekt dar. Zusammen mit der reformierten ENP böte die Östliche Partnerschaft neue Anreize für die Partnerstaaten (,market, mobility, money'). Priesmeyer-Tkocz äußerte Kritik daran, dass die Östliche Partnerschaft unter der Heterogenität der Interessen leide, sowohl zwischen den Mitgliedstaaten der Europäischen Union als auch unter den Adressaten. Nicht alle Mitgliedstaaten seien gleichsam an Beziehungen mit den osteuropäischen Staaten interessiert. Ferner seien auch nicht alle Adressaten bereit, sich zu den europäischen Werten der Rechtsstaatlichkeit, Demokratie und guten Regierungsführung im gleichen Maße zu bekennen.

Die im Rahmen der Konferenz dargestellte Vielseitigkeit und Vielschichtigkeit der strate- 
gischen Ansätze verdeutlicht die Gewichtung der außenpolitischen Beziehungen ebenso wie ihre Komplexität. Es besteht eine Vielzahl von Kooperationsmöglichkeiten, die unterschiedlich erfolgreich und effizient sind. Eckart Stratenschulte fasste dieses Konstrukt sowie die Problematik der zukünftigen Entwicklung tref- fend zusammen, indem er betonte, dass es zwei essenzielle Fragen gebe. Zum einen wohin die Reise gehen soll und zum anderen, wer mitfahren soll. Die Problematik der strategischen Ansätze der Europäischen Union besteht darin, zu entscheiden, welche dieser Fragen zuerst beantwortet wird.

\section{Rechte türkischer Staatsangehöriger in der EuGH-Rechtsprechung}

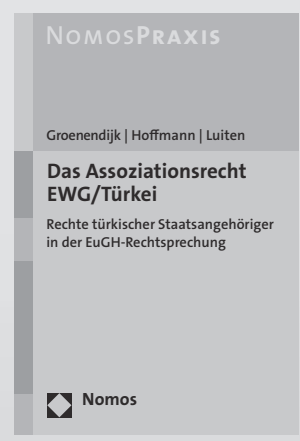

Das Assoziationsabkommen und die Assoziationsratsbeschlüsse der Europäischen Gemeinschaft mit der Türkei, sind seit den Entscheidungen „Demirel" und „Sevince" von erheblicher und

\section{Das Assoziationsrecht EWG/Türkei}

Rechte türkischer Staatsangehöriger in der EuGH-Rechtsprechung Von Kees Groenendijk, Holger Hoffmann und Maaike Luiten 2013, 127 S., brosch., 34,- $€$ ISBN 978-3-8487-0056-1

ständig wachsender Bedeutung für die europarechtliche Praxis. Sie sind Teil des EU-Rechts und unmittelbar anwendbar. 